\title{
A Planar Reconfigurable Radiation Pattern Dipole Antenna with Reflectors and Directors for Wireless Communication Applications
}

\author{
Imen Ben Trad, ${ }^{1,2}$ Jean Marie Floc'h, ${ }^{2}$ Hatem Rmili, ${ }^{3}$ \\ M'hamed Drissi, $^{2}$ and Fethi Choubani ${ }^{1}$ \\ ${ }^{1}$ INNOV'COM, Sup'Com, University of Carthage, 2083 Ariana, Tunisia \\ ${ }^{2}$ IETR, INSA, 20 Avenue Buttes des Coësmes, 35043 Rennes, France \\ ${ }^{3}$ Electrical and Computer Engineering Department, Faculty of Engineering, King Abdulaziz University, P.O. Box 80204, \\ Jeddah 21589, Saudi Arabia
}

Correspondence should be addressed to Hatem Rmili; hmrmili@kau.edu.sa

Received 27 August 2013; Revised 5 December 2013; Accepted 6 December 2013; Published 5 January 2014

Academic Editor: Zhongxiang Shen

Copyright (c) 2014 Imen Ben Trad et al. This is an open access article distributed under the Creative Commons Attribution License, which permits unrestricted use, distribution, and reproduction in any medium, provided the original work is properly cited.

\begin{abstract}
A planar printed dipole antenna with reflectors and directors, able to steer its radiation pattern in different directions, is proposed for telecommunication applications. Starting from a dual-beam printed dipole antenna achieved by combining two elementary dipoles back to back, and by loading four PIN diodes, three modes of reconfigurable radiation patterns are achieved at the frequency $2.56 \mathrm{GHz}$ thanks to switches states. A prototype of the structure was realized and characterized; an efficiency of $75 \%$ is obtained. Simulation and measured results of the results are presented and discussed.
\end{abstract}

\section{Introduction}

In the latest years, reconfigurable radiation pattern antennas are becoming more and more desired because of the increasing demands on the quality and capacity of the telecommunication systems. The capability to steer the beam's directions electronically extends the functionalities of the antennas and adds more flexibility. In addition, the radiation pattern agility characteristic contributes on avoiding noisy environments, improving security, and saving energy by better directing signal toward intended users which enhance the overall performances of the modern wireless communication systems.

Several reconfigurable radiation pattern antennas have been reported such as a three-layer switchable radiation pattern antenna based on the conventional Yagi antenna [1], an hexagonal cylinder antenna with moderate gain of $5 \mathrm{dBi}$ [2], and a central circular patch with two paddle-shaped parasitic patches [3].

Among many types of reconfigurable antennas, planar structures [4-8] in particular have been extensively investigated because of their attractive features such as simple structure, low profile, light weight, and ease of fabrication and integration.

In [4], a printed planar ring-dipole microstrip fed antenna was designed to switch its radiation pattern in different directions depending on the state of six loaded PIN diode switches. A planar slotted bow-tie antenna with reconfigurable pattern properties was reported in [5]; the antenna consists of a pair of reconfigurable CPW-to-slot line transitions, a pair of Vivaldi-shaped radiating tapered slots, and four PIN diodes. The compact wideband antenna with alternate reflector and radiator presented in [6] achieves two directional radiation patterns by using short and open circuits (The maximum gain obtained is about $4.5 \mathrm{dBi}$ for the RRC and about $-5 \mathrm{dBi}$ for the LRC).

In this paper, a planar printed dipole antenna with reconfigurable radiation performances operating at $2.56 \mathrm{GHz}$ was designed. Depending on the states of four loaded PIN diodes, the antenna can select between three modes. In the first mode Ml, a dual-beam can be achieved in both 


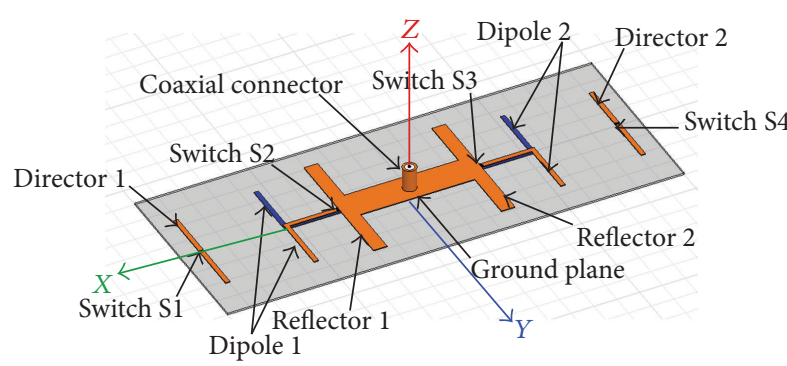

(a)

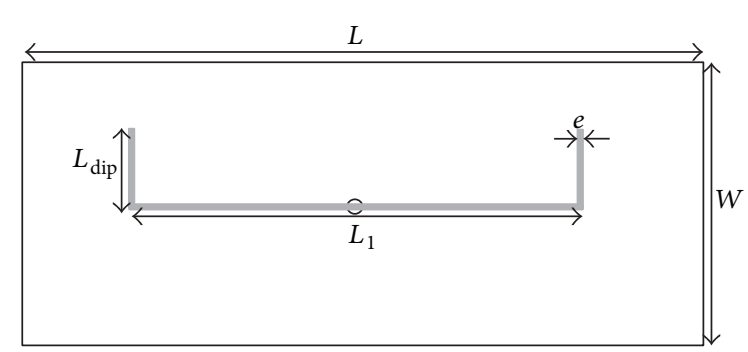

(b)

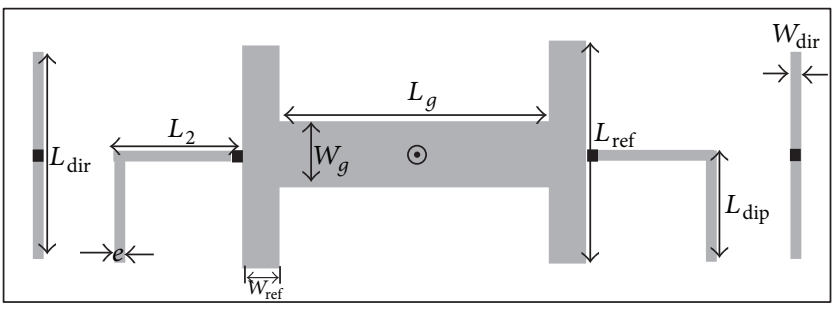

(c)

FIGURE 1: (a) Schema of the reconfigurable dipole antenna: (b) top view; (c) back view.

$x$ - and $-x$-directions by turning $\mathrm{ON}$ all the RF switches, and a measured peak gain of $7.3 \mathrm{dBi}$ is well reached. In the second mode M2, when only one dipole and its director are enabled, a unique directive beam can be radiated in $x$ - or $-x$-directions with a half-power beamwidth (HPBW) of $48^{\circ}$ and a measured peak gain of $8.12 \mathrm{dBi}$. Finally by deactivating the director in the second mode M2, we may obtain the third mode M3. Therefore, when only the elementary dipole is activated, a less directive pattern would be reached with larger HPBW $\left(94^{\circ}\right)$ and a lower measured peak gain $(5.37 \mathrm{dBi})$. The designed structure presents a measured peak efficiency of $75 \%$ at the operating frequency.

\section{Antenna Design}

The planar dual-beam dipole antenna was performed to operate at $2.45 \mathrm{GHz}$ by printing two elementary dipoles (denoted dipole 1 and dipole 2) back to back on an Arlon CuClad217 substrate of thickness $h=0.8 \mathrm{~mm}$ and relative permittivity $\varepsilon_{r}=2.17$. As it is shown in Figure 1 , two identical dipole arms of dimension $\left(\left(L_{1} / 2\right) \times L_{\text {dip }}\right)$ were printed on the top layer of the substrate, whereas, on the bottom side, two identical reflectors $\left(L_{\text {ref }} \times W_{\text {ref }}\right)$, two identical directors $\left(L_{\text {dir }} \times\right.$ $\left.W_{\text {dir }}\right)$, a ground plane $\left(L_{g} \times W_{q}\right)$, and the two other dipole arms $\left(L_{2} \times L_{\text {dip }}\right)$ were printed. A prototype was manufactured and characterized, and measurements were compared to simulation results [9]. Then, in order to electronically control the radiation pattern of the dual beam antenna, four PIN diodes were loaded on the bottom side. Two diodes were integrated between each reflector and the dipole arm, while the two others were implanted in the middle of the two directors as depicted in Figure 1.

The design parameters are summarized in Table 1. Depending on the PIN diode states, three main modes of radiation were obtained for the reconfigurable structure.
TABLE 1: Design parameters.

\begin{tabular}{lc}
\hline Parameter & Dimension $(\mathrm{mm})$ \\
\hline$L$ & Value \\
$W$ & 209 \\
$L_{\text {dip }}$ & 62 \\
$L_{1}$ & 27.5 \\
$L_{\text {dir }}$ & 116 \\
$W_{\text {dir }}$ & 45 \\
$L_{\text {ref }}$ & 2 \\
$W_{\text {ref }}$ & 60 \\
$e$ & 6 \\
$W_{g}$ & 2 \\
$L_{2}$ & 14 \\
$L_{g}$ & 27 \\
\hline
\end{tabular}

These modes are detailed in Table 2. Mode M1 is defined when all the switches are activated in order to compare the active structure with the passive one (without switches). Mode M2 represents the operation of one compartment (dipole + reflector + director) in the structure when the other is deactivated. Mode M3 excludes the effect of the director in one compartment described in mode M2. We have omitted to consider mode 4 where both of the dipoles are deactivated because it is with no interest since the structure is not excited at all.

The implementation procedure of the PIN diodes is complex. That is why we decided to load only two PIN diodes between each reflector and the dipole arm as shown in Figure 2 to prove the concept. Then, short and open circuits will be used for the two directors for the rest of measurements. 
TABLE 2: Mode configurations by switches on/off conditions.

\begin{tabular}{lcccccc}
\hline Mode & Configuration & State & Switch 1 & Switch 2 & Switch 3 & Switch 4 \\
\hline Mode 1 & All dipoles and directors are activated. & S1 & On & On & On & On \\
\hline \multirow{2}{*}{ Mode 2 } & Only dipole 1 and director 1 are activated. & S21 & On & On & Off & Off \\
& Only dipole 2 and director 2 are activated. & S22 & Off & Off & On & On \\
\hline \multirow{2}{*}{ Mode 3 } & Only dipole 1 is activated. & S31 & Off & On & Off & Off \\
& Only dipole 2 is activated. & S32 & Off & Off & On & Off \\
\hline
\end{tabular}

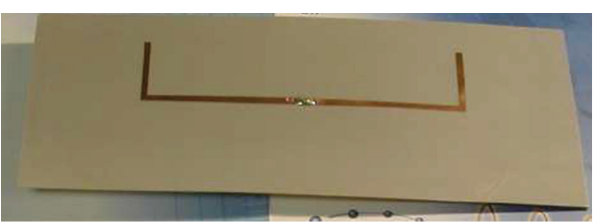

(a)

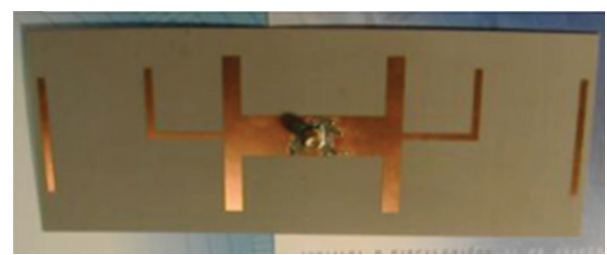

(b)

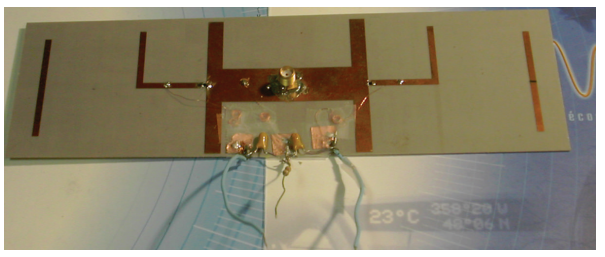

(c)

FIgure 2: Photo of the prototyped antennas: The passive structure (a) top view; (b) back view; (c) with the integrated PIN diodes and the related bias circuit.

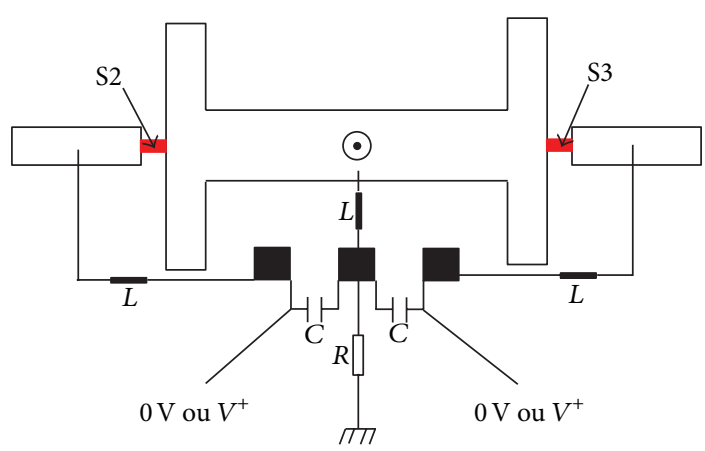

FIgURE 3: Bias circuit of the PIN diodes.

The used Skyworks-SMP 1320-079LF diodes were chosen for their low series resistance.

To control the status of the diodes, a bias circuit composed of three pads (two were connected to the positive terminal of the power supply), three chokes, and a resistance $R=1 \mathrm{k} \Omega$ connected to the power supply ground was used, but it does not affect the behavior of the antenna. To isolate the dc while maintaining continuity for the RF, two capacitors $(C=10 \mathrm{nF})$ were integrated to the bias network as shown in Figure 3. The supplied control voltage was about $V^{+}=1.5 \mathrm{~V}$.

In simulation, the diodes were modeled as short and open circuits; losses were not taken into consideration.

\section{Results and Discussion}

The reconfigurable radiation pattern antenna was simulated using the 3D electromagnetic simulator Ansoft-HFSS 13, realized and experimentally characterized at the operating frequency $2.56 \mathrm{GHz}$.

The realized prototype was investigated by measuring its impedance matching over the frequency band $2-3 \mathrm{GHz}$ and its radiation properties (gain, efficiency, and radiation patterns) by using an anechoic room (Satimo Stargate from the IETR Institute). Good agreement between measured and simulated results is obtained. The implantation of switches as described above allows the electronic control of the radiated beam at the operating frequency by activating or deactivating one elementary dipole and/or directors.

In order to better understand the radiation mechanism of the structure, we have analyzed simulated surface current distributions inside the antenna and the $2 \mathrm{D} / 3 \mathrm{D}$ radiation patterns for all the considered modes.

In this paragraph, we present simulated and measured results as impedance matching, surface current distribution, radiation patterns, gain, and efficiency dealing with all modes. For mode M1, we show main results, whereas for modes M2 and M3, and due to the symmetry of the structure, we chose to present, in addition to simulations, only measured results dealing with mode M2/state S21 and mode M3/state S3, where the antenna radiates in the $x$ direction. 


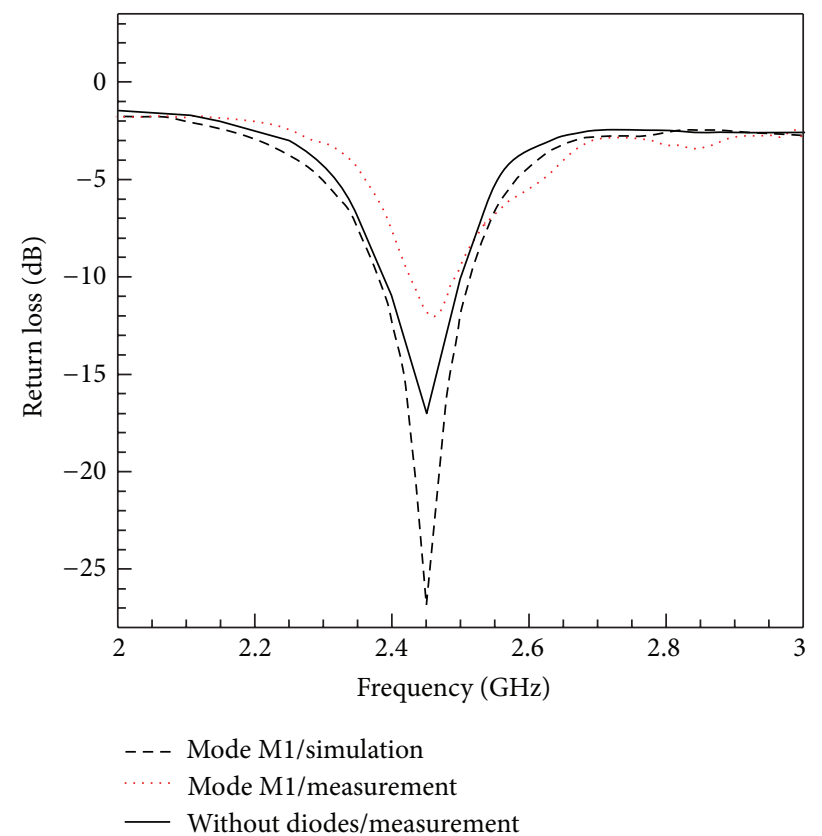

Figure 4: Simulated and measured return loss of the antenna (mode M1) at the resonant frequency $2.45 \mathrm{GHz}$ without diodes, and when all diodes are $\mathrm{ON}$.
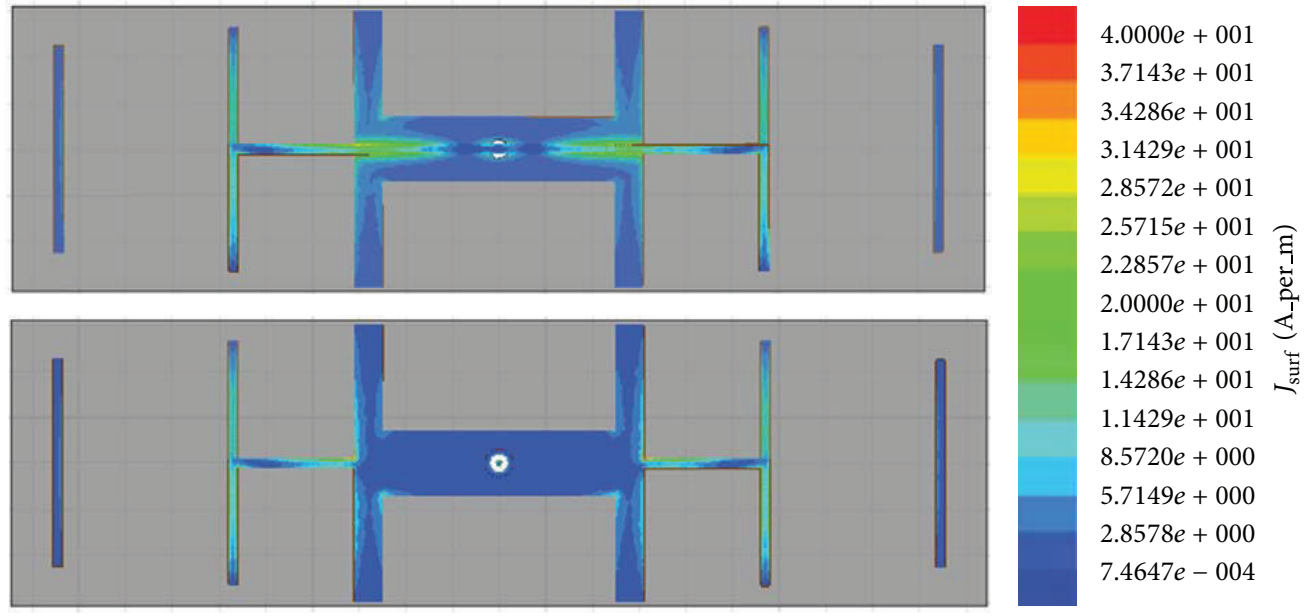

(a)

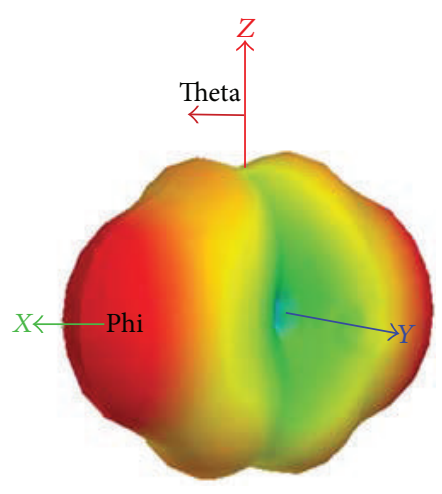

(b)

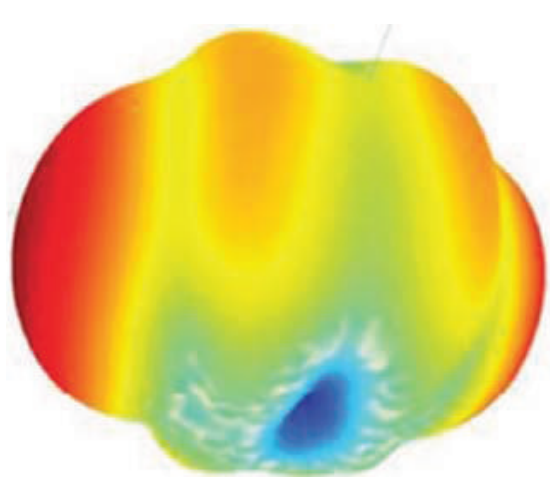

(c)

Figure 5: The dual-beam dipole antenna (in mode M1) at the resonant frequency $2.45 \mathrm{GHz}$ : (a) Simulated current distribution; (b) 3DSimulated radiation pattern; (c) 3D-measured radiation pattern. 


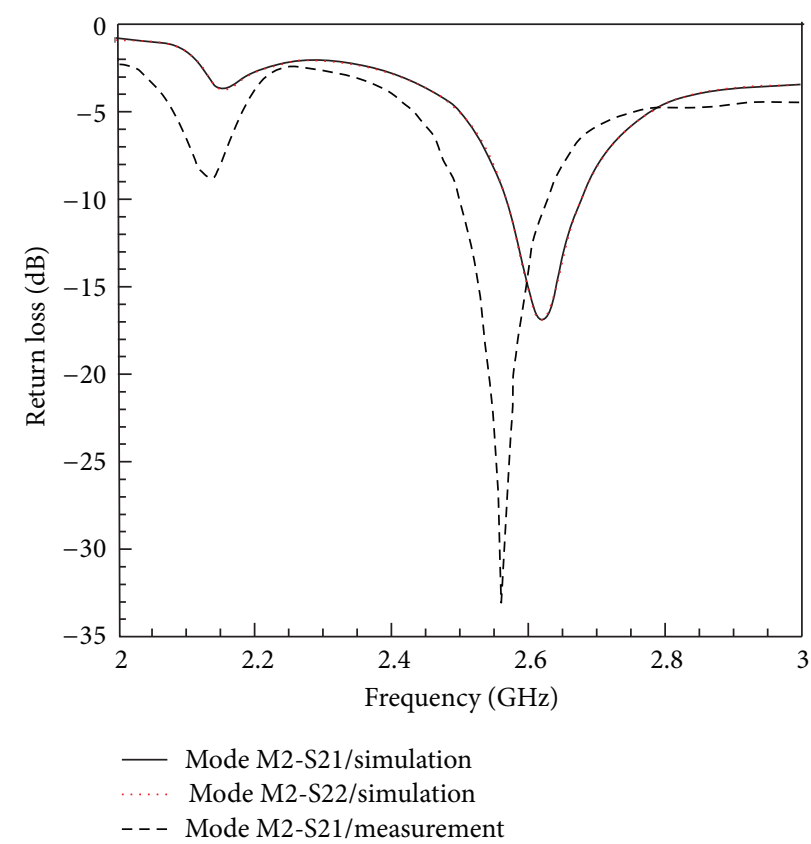

FIGURE 6: Simulated and measured return loss of the antenna when operating in Mode M2.

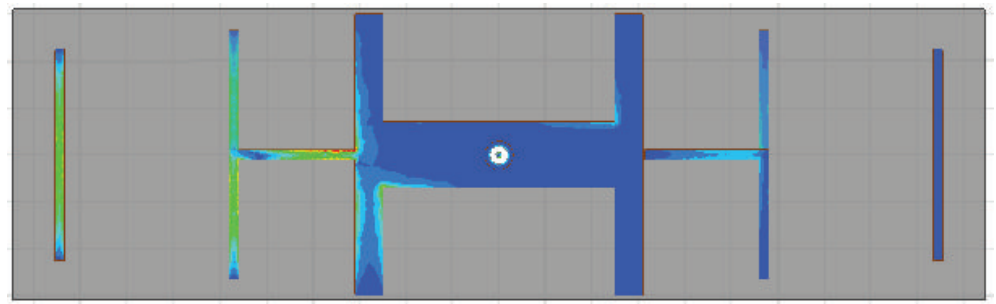

(a)

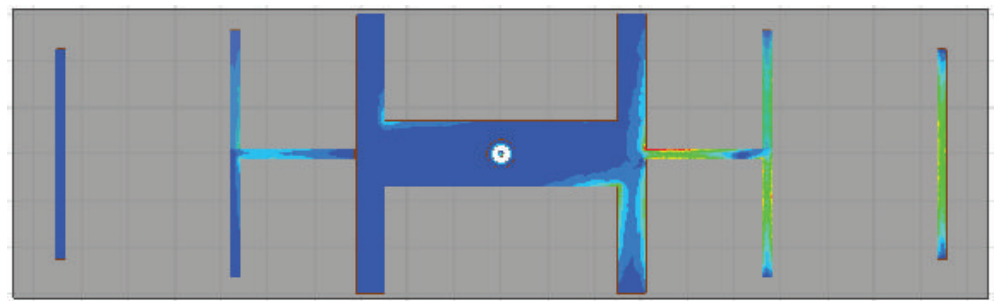

(b)

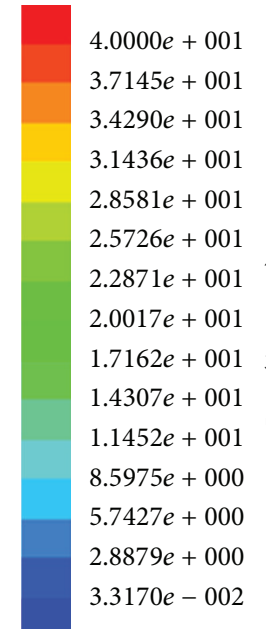

\section{.

FIGURE 7: Simulated current distributions at the operating frequency 2.63 GHz for mode M2: (a) S21-State; (b) S22-State.

3.1. Mode M1: Dual-Beam Pattern. When PIN diodes are loaded and are turned ON, the dipole is still able to operate at the operating frequency $2.45 \mathrm{GHz}$ as it can be noticed from Figure 4 which exhibits simulated and measured return losses of the starting dipole with and without switches. We can conclude that the effect of the bias circuit on the antenna operation is week.

As it is well known, by placing a reflector (whose length should be larger than the dipole length: $L_{\text {ref }} \approx 1.1 \times 2 L_{\text {dip }}$ ) near the dipole antenna, an eccentric pattern will be reached with a maximum radiation towards the dipole. The addition of a director (whose length should be lower than the dipole length: $L_{\mathrm{dir}} \approx 0.8 \times 2 L_{\mathrm{dip}}$ ) allows for the improvement of the gain by better directing the radiated beams. According to
Figure 5(a), when all RF switches are disconnected (passive antenna) or at $\mathrm{ON}$ state, the two elementary dipoles radiate. Moreover, as it can be seen in Figures 5(b) and 5(c), simulated and measured radiation patterns show a dual-beam behavior, where the maximum gain (around $7 \mathrm{dBi}$ ) was obtained in the $x$-direction (axis of the dipoles), as it was expected.

3.2. Mode M2: High Directive Reconfigurable Beam. When mode M2 is excited, the resonance frequency of the dipole shifts slightly to higher frequencies. As it is shown in Figure 6, the simulated operating frequency is $2.63 \mathrm{GHz}$, whereas the measured one is centered around $2.56 \mathrm{GHz}$. This frequency shift may be explained by the possible effect of the bias circuit. 


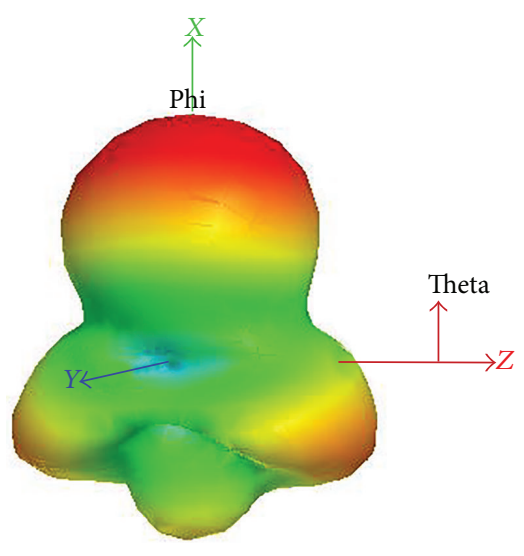

(a)

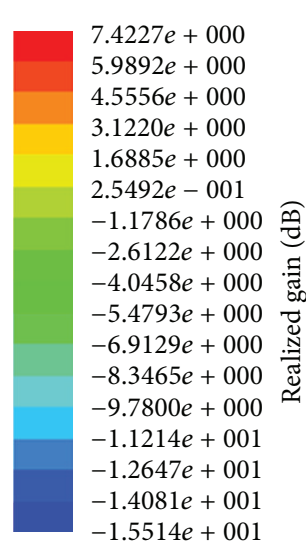

$-1.4081 e+001$
$-1.5514 e+001$

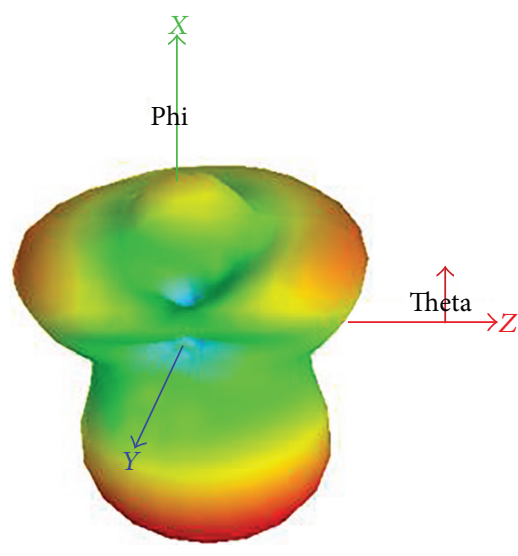

(b)

FIGURE 8: 3D-simulated radiation patterns at 2,63 GHz for mode M2: (a) S21-State; (b) S22-State.

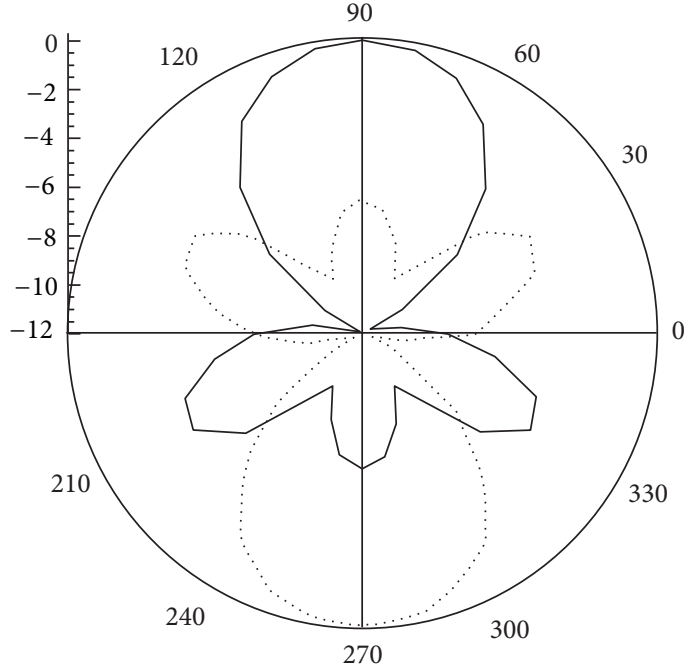

Mode M2/state-S21
$\ldots . .$. Mode M2/state-S22

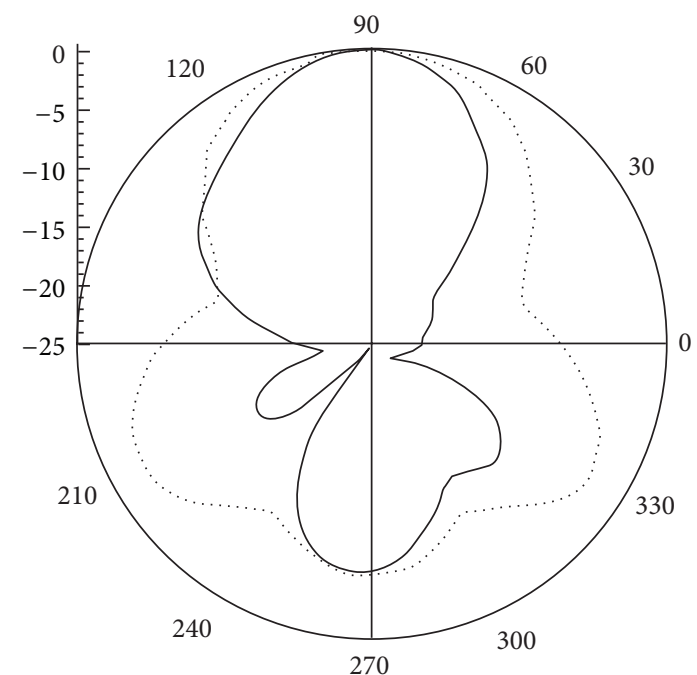

Mode M2/state-S21/simulation Mode M2/state-S21/measurement

(b)

Figure 9: 2D-simulated radiation patterns for mode M2 (S21- and S22-states) at 2.63 GHz: (a) 2D-simulated (at 2.63 GHz) and measured (at $2,56 \mathrm{GHz}$ ) radiation patterns for S21-State of Mode M2.

For state S21 of mode M2, the surface current distribution presented in Figure 7(a) shows that dipole 2 and director 2 are disabled, and only dipole 1 and director 1 radiate in the $x$-direction. Measured 2D-radiation patterns confirm this behavior predicted by simulations as shown in Figures 8 and 9.

The radiation in $-x$-direction is simply obtained by activating dipole 2 and deactivating dipole 1; the structure operates then in S22-state of mode M2.

As it can be noticed from Figures 8 and 9, we can remark the presence of a rear radiation for S21- and S22-states.

Because of the symmetrical structure, the reconfigurable dipole antenna presents the same performances in both $x$ - and $-x$-directions. In both cases, the measured HPBW is around $48^{\circ}$. The observed asymmetrical measured 2Dpattern may be attributed to diodes and their associated bias circuits.

Figure 10 exhibits the measured gain evolution over the frequency band $2,4-2,7 \mathrm{GHz}$ (centered on the resonant frequency $2,56 \mathrm{GHz}$ ) for the reconfigurable antenna when operating state S21 of mode M2. The reconfigurable radiation pattern antenna presents a simulated peak gain of $7.42 \mathrm{dBi}$ at the resonant frequency $2.63 \mathrm{GHz}$, whereas the measured maximum gain is $8.12 \mathrm{dBi}$ at the operating frequency $2.56 \mathrm{GHz}$. It is worth to know that the measured maximum gain for the passive structure is $7.3 \mathrm{dBi}$. 


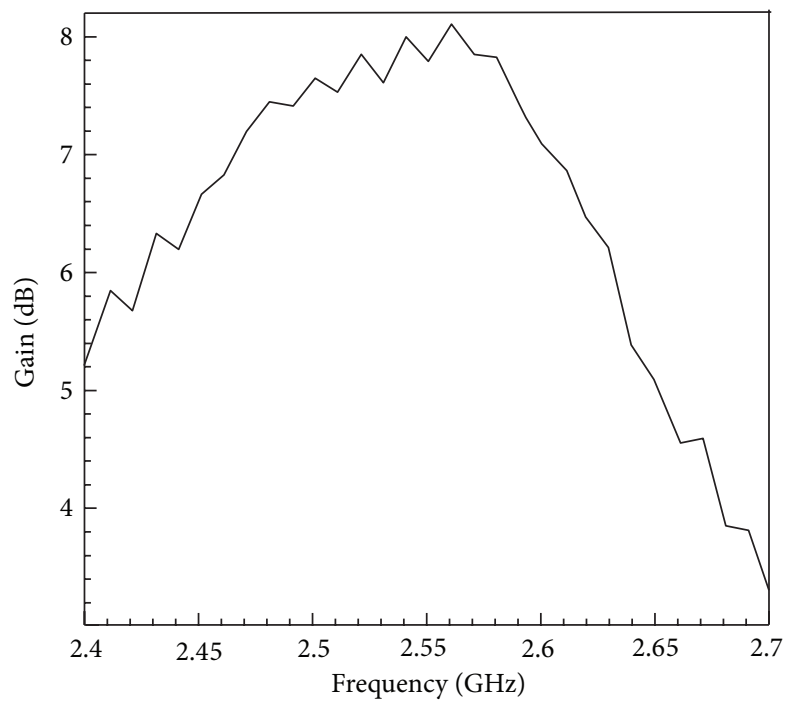

FIGURE 10: Measured gain of the reconfigurable antenna when operating in S21-state of mode M2.

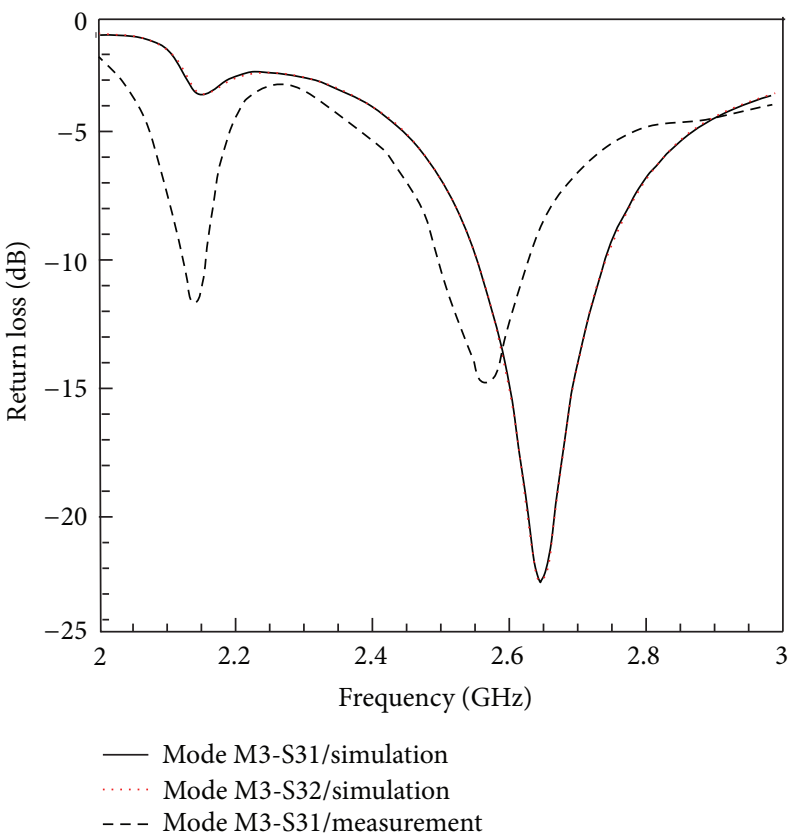

FIGURE 11: Simulated and measured return loss of the dipole when operating in Mode M3.

3.3. Mode M3: Less Directive Reconfigurable Beam. As for mode M2, when mode M3 is excited, the operating frequency of the reconfigurable dipole moves slowly to higher frequencies. Figure 11 shows a measured resonance frequency of $2.56 \mathrm{GHz}$.

When S31 state is active (we keep switch S2 ON and we turn OFF switches S1, S3, and S4), dipole 2 will be obviously deactivated as explained above (S21 state of mode M2) and the two strips of the director 1 will be disconnected; thus, the surface current distribution will be altered and director 1 becomes invisible for dipole 1 as depicted in Figure 12(a). Then, the antenna radiates in the $x$ direction.

Otherwise, if we excite S32 state as shown in Figure 12(b), the antenna will radiate fairly as described for S22 state of the mode M2 (presented in Figure $7(\mathrm{~b})$ ); the radiation pattern will be then oriented in the $-x$-direction; however, director 2 (not considered as a single strip when S4 is turned Off) becomes unseen for dipole 2 which explains the decrease of the measured gain of nearly $2.75 \mathrm{dBi}$ compared to both of the S21 and S22-states of mode M2 presented earlier as depicted in Figures 13 and 14. 


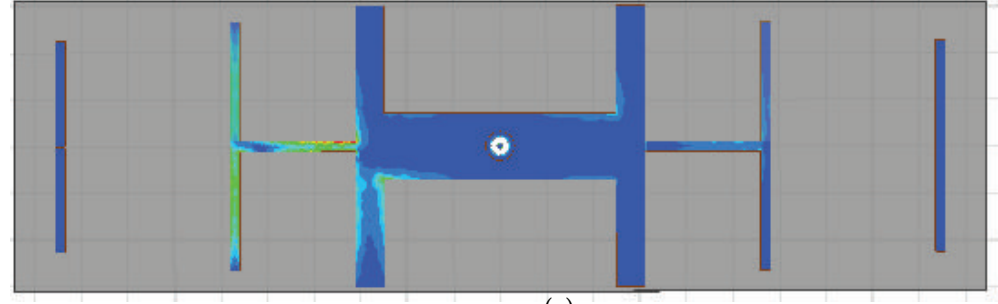

(a)

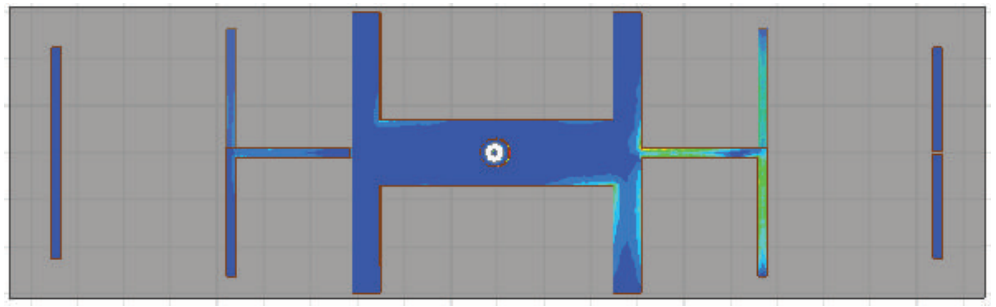

(b)

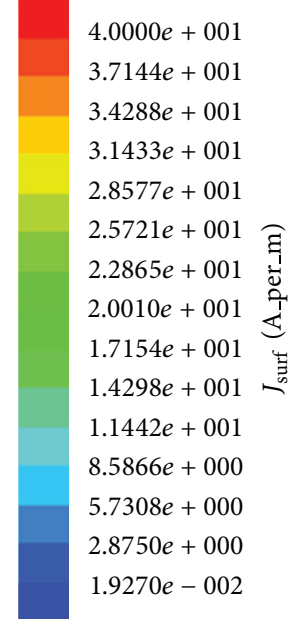

FIGURE 12: Simulated current distributions at the operating frequency $2.64 \mathrm{GHz}$ for mode M3: (a) S31-State; (b) S32-State.

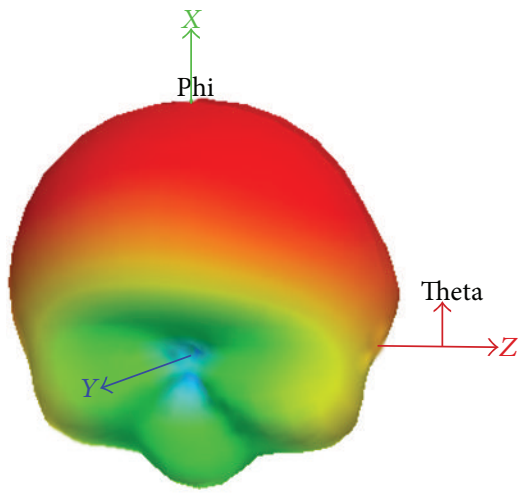

(a)

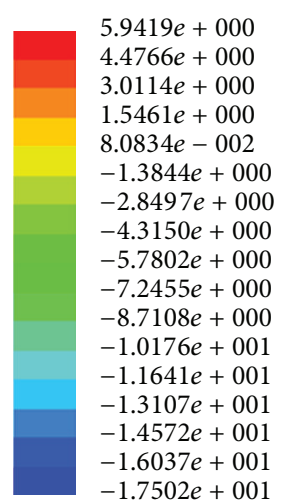

$-1.7502 e+001$

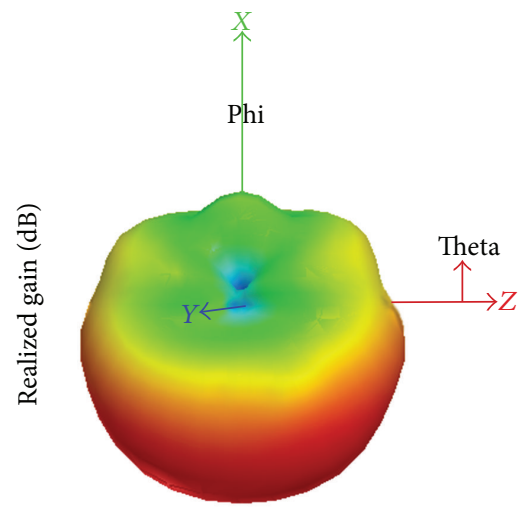

(b)

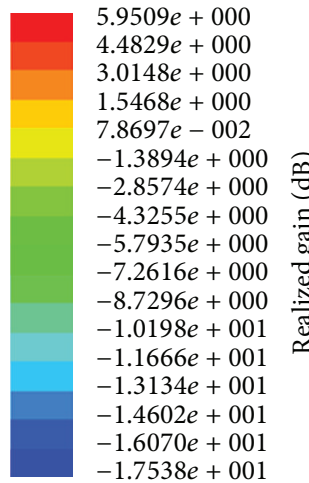

$-1.7538 e+001$

FIGURE 13: 3D-simulated radiation patterns at 2,64 GHz for mode M3: (a) S31-State; (b) S32-State.

By comparison with mode M2, we notice that for both S31 and S32 states, the rear radiation of the antenna is significantly reduced, the radiation patterns become then less directive with larger HPBW (the measured value is $94^{\circ}$ ). Besides, the measured 2D-radiation pattern matches well with simulations.

It is trivial to note that, for modes M2 and M3, when the elementary dipole is disabled, turning $\mathrm{ON}$ or OFF switches (S1 or S4) loaded on the corresponding director is with no interest (the director will be consequently disabled).

As mentioned before, because of the symmetry of the antenna, the dipole behaves in the same manner in both $x$-direction and $-x$-direction. That is why, it is sufficient to study the state $\mathrm{S} 31$ of the mode $\mathrm{M} 3$ (when the antenna radiates in $x$-direction). The deactivation of director 1 in addition to the dipole 2 leads to a less directive beam in the $x$-direction with a simulated gain of $5.9 \mathrm{dBi}$. The measured gain is around $5.37 \mathrm{dBi}$ as it can be deduced from Figure 15 .
TABLE 3: Summarized measured peak gain and maximum beam direction.

\begin{tabular}{lcccc}
\hline & State & $\begin{array}{c}\text { Beam's } \\
\text { direction }\end{array}$ & $\begin{array}{c}\text { Half-power } \\
\text { beamwidth } \\
\left({ }^{\circ}\right)\end{array}$ & $\begin{array}{c}\text { Max gain } \\
(\mathrm{dBi})\end{array}$ \\
\hline Mode M1 & S1 & $-x$ and $+x$ & - & 7.3 \\
\hline \multirow{2}{*}{ Mode M2 } & S21 & $+x$ & 48 & 8.12 \\
& S22 & $-x$ & 48 & 8.12 \\
\hline \multirow{2}{*}{ Mode M3 } & S31 & $+x$ & 94 & 5.37 \\
& S32 & $-x$ & 94 & 5.37 \\
\hline
\end{tabular}

3.4. Summary. One can see that, as summarized in Table 3, according to the PIN diodes states, the antenna can switch its radiation pattern in both $x$ - and $-x$-directions. At the same time, it can move from a directive radiation pattern to a less directive one (since the corresponding director, which 


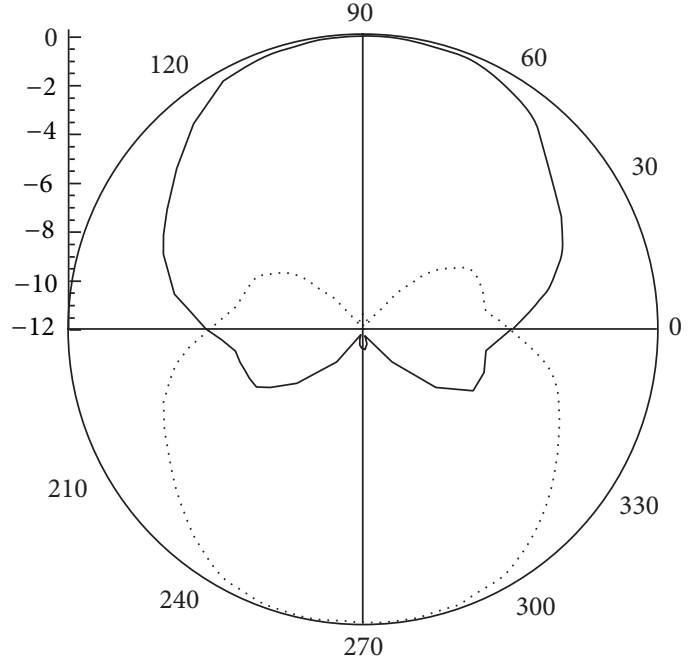

Mode M3/state-S31 Mode M3/state-S32

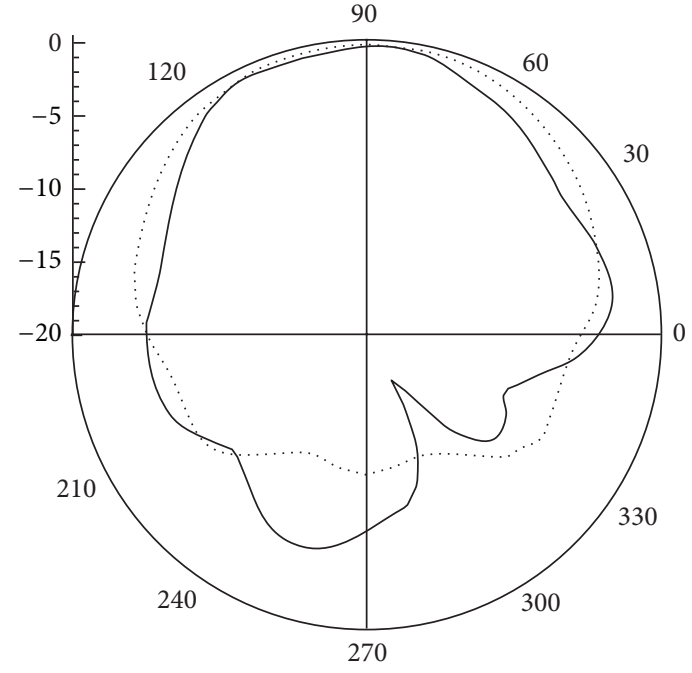

Mode M3/state-S31/simulation Mode M3/state-S31/measurement

(b)

FIGURE 14: (a) 2D-simulated radiation patterns for mode M3 (S31- and S32-states) at 2,64 GHz: (a) 2D-simulated (at 2.64 GHz) and measured (at $2.56 \mathrm{GHz}$ ) radiation patterns for S31-State of Mode M3.

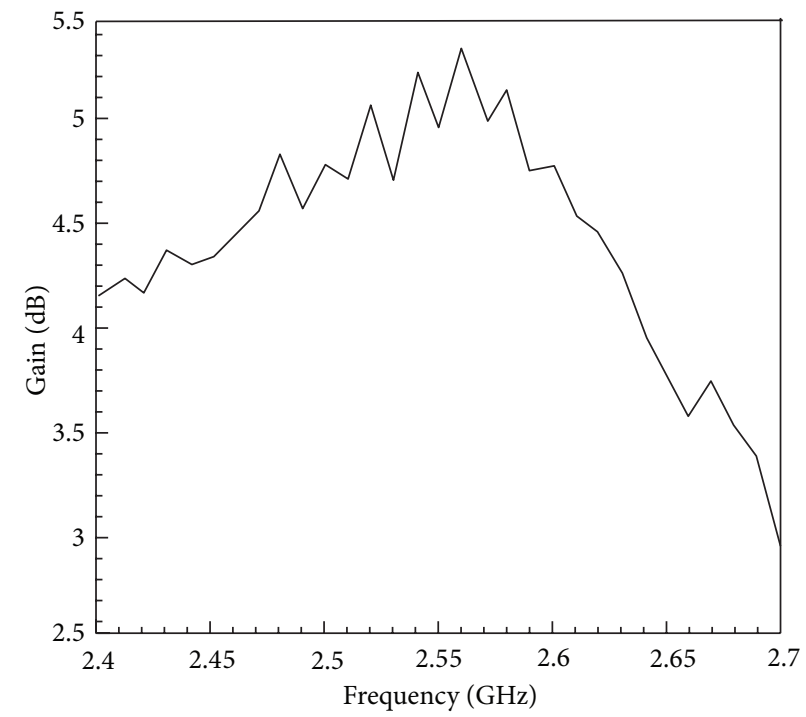

FIGURE 15: Measured gain of the reconfigurable antenna when operating in S31-state of mode M3.

naturally improves the gain by better directing the radiation, was disabled).

As it can be seen in Figure 16, the active antenna is slightly less efficient than the passive one but still able to achieve a measured efficiency of $75 \%$ at the operating frequency.

The integration of RF switches and their related bias circuits improves the antenna performances, while keeping the antenna as efficient as the passive structure.

\section{Conclusion}

A planar printed dipole antenna, designed by combining two elementary dipoles, with reconfigurable radiation pattern properties has been proposed for wireless communication systems. The agility was ensured by using four PIN diodes. The antenna which operates around $2,56 \mathrm{GHz}$ is able to tilt its radiation pattern in both $-x$ - and $x$-directions depending on the switch states. We can obtain in one mode a directive 


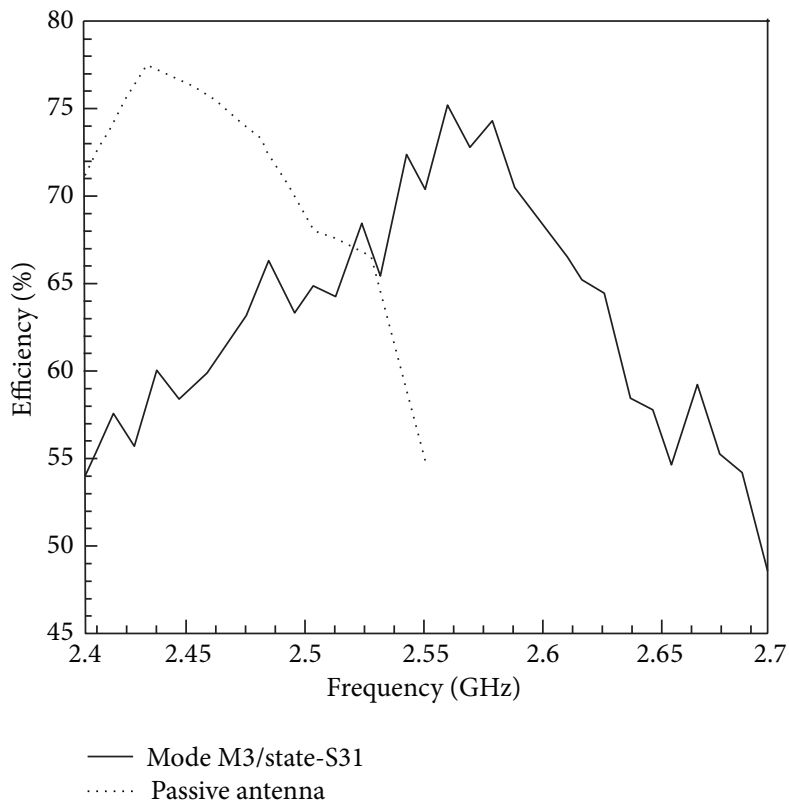

Figure 16: Measured efficiency of both passive, and reconfigurable (S31-state of mode M3) antennas.

pattern with $8.12 \mathrm{dBi}$ of gain and a less directive pattern with $5.37 \mathrm{dBi}$ in another mode. If all switches are turned $\mathrm{ON}$, the antenna founds its dual beam radiation at $2.45 \mathrm{GHz}$. One can also tune the operating frequency of the antenna by adjusting the distance between dipoles and reflectors, the distance between the two reflectors, and the size of the reflectors.

\section{Conflict of Interests}

The authors declare that there is no conflict of interests regarding the publication of this paper.

\section{Acknowledgment}

This paper was funded by the Deanship of Scientific Research (DSR), King Abdulaziz University, Jeddah. The authors, therefore, acknowledge with thanks DSR technical and financial support.

\section{References}

[1] J. Sahaya kulandai Raj, J. Fahlbusch, and J. Schoebel, "A beam switching three layer reconfigurable antenna," in Proceedings of the 7th German Microwave Conferance (GeMiC '12), pp. 1-4, Ilmenau, Germany, March 2012.

[2] Y. L. Tsai, R. B. Hwang, and Y. D. Lin, "A reconfigurable beamswitching antenna base on active fSS," in Proceedings of the International Symposium on Antenna Technology and Applied Electromagnetics (ANTEM '12), pp. 1-4, Toulouse, France, June 2012.

[3] X. Cai, A. Wang, W. Leng, and J. Pei, "Novel radiation pattern reconfigurable antenna using shorting probe," in Proceedings of the International Conference on Electronics, Communications and Control (ICECC '11), pp. 2727-2730, Zhejiang, China, September 2011.
[4] I. Ben Trad, J. M. Floc'h, H. Rmili, M. Drissi, and F. Choubani, "Design of reconfigurable radiation pattern ring-dipole antenna for wireless communication," in Proceedings of the 7th European Conference on Antennas and Propagation (EuCAP '13), pp. 12521255, Gothenburg, Sweden, April 2013.

[5] S.-J. Wu and T.-G. Ma, "A wideband slotted bow-tie antenna with reconfigurable CPW-to-slotline transition for pattern diversity," IEEE Transactions on Antennas and Propagation, vol. 56, no. 2, pp. 327-334, 2008.

[6] G. M. Zhang, J. S. Hong, G. Song, and B. Z. Wang, "Design and analysis of a compact wideband pattern-reconfigurable antenna with alternate reflector and radiator," IET Microwaves, Antenna and Propagation, vol. 6, no. 15, pp. 1629-1635, 2012.

[7] W. Kang, S. Lee, and K. Kim, "Design of symmetric beam pattern reconfigurable antenna," Electronics Letters, vol. 46, no. 23, pp. 1536-1537, 2010.

[8] D. Patron, D. Piazza, and K. R. Dandekar, "Wideband planar antenna with reconfigurable omni directional and directional patterns," Electronics Letters, vol. 49, no. 8, pp. 516-518, 2013.

[9] J. M. Floc'h, A. El Sayed Ahmad, and Y. Kokar, "Dual-beam antenna design for autonomous sensor network applications," International Journal of Antennas and Propagation, vol. 2012, Article ID 289681, 7 pages, 2012. 

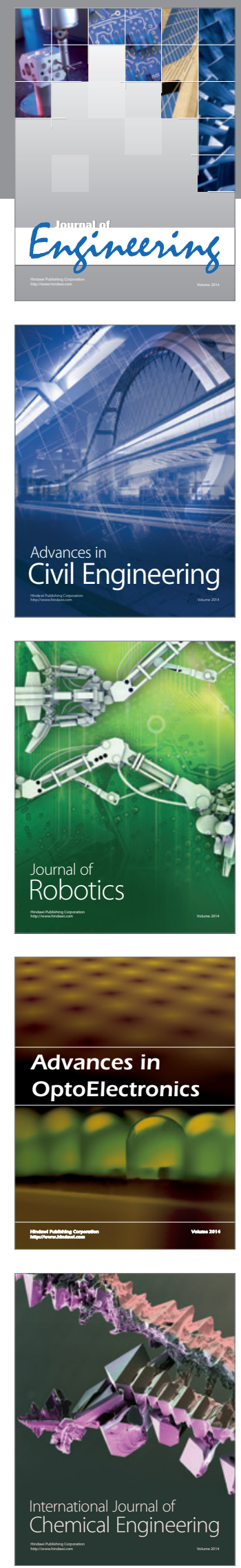

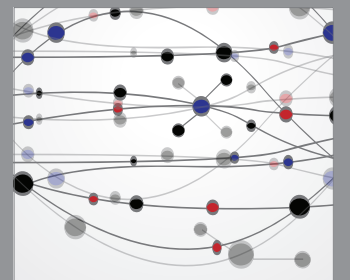

The Scientific World Journal
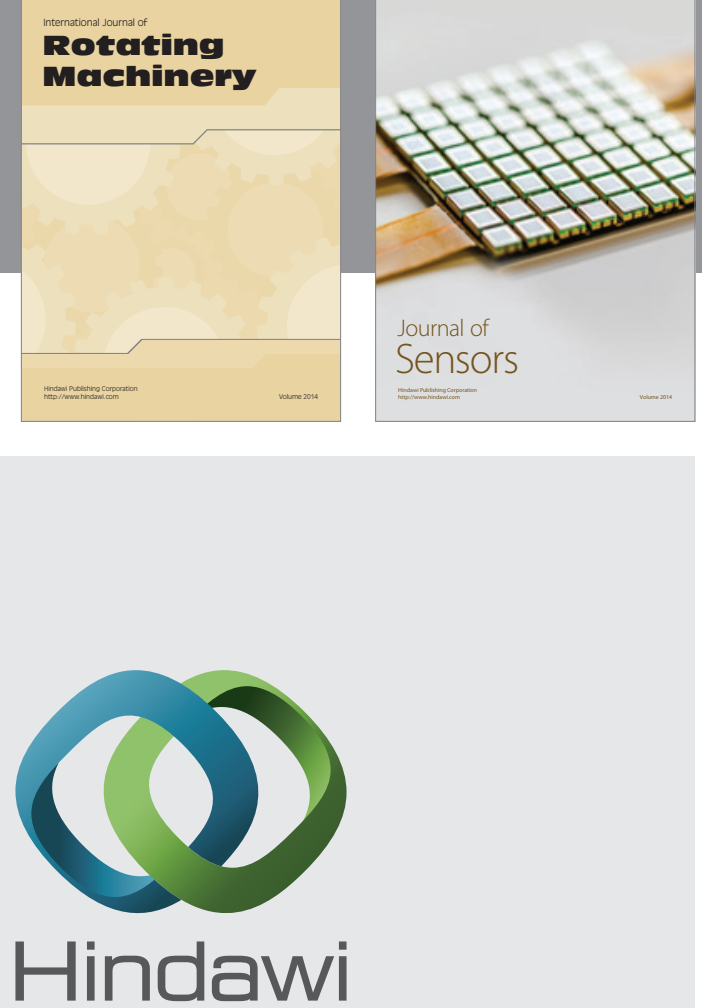

Submit your manuscripts at http://www.hindawi.com
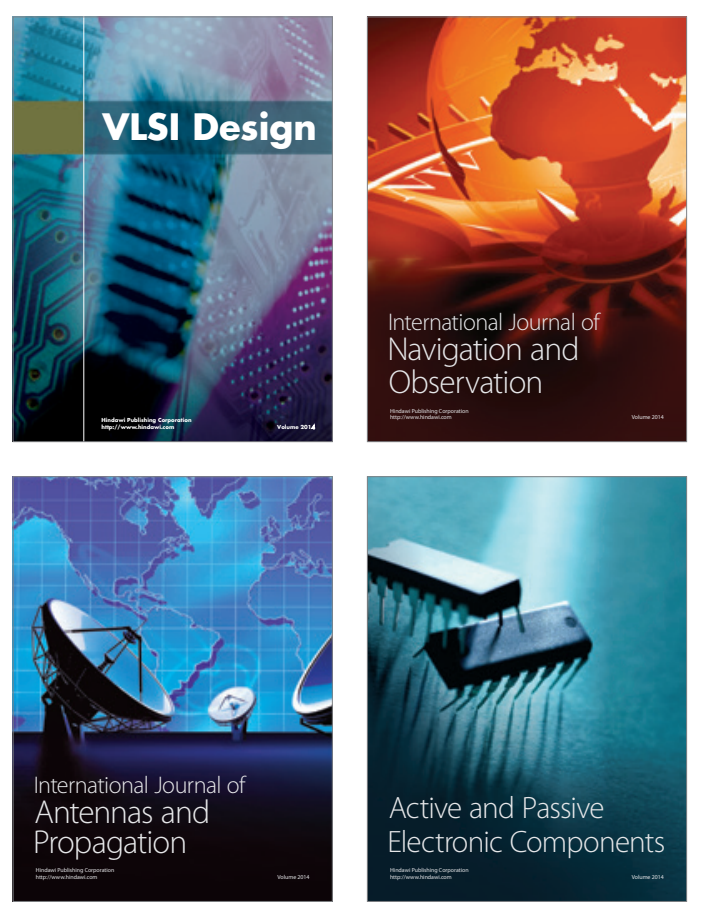
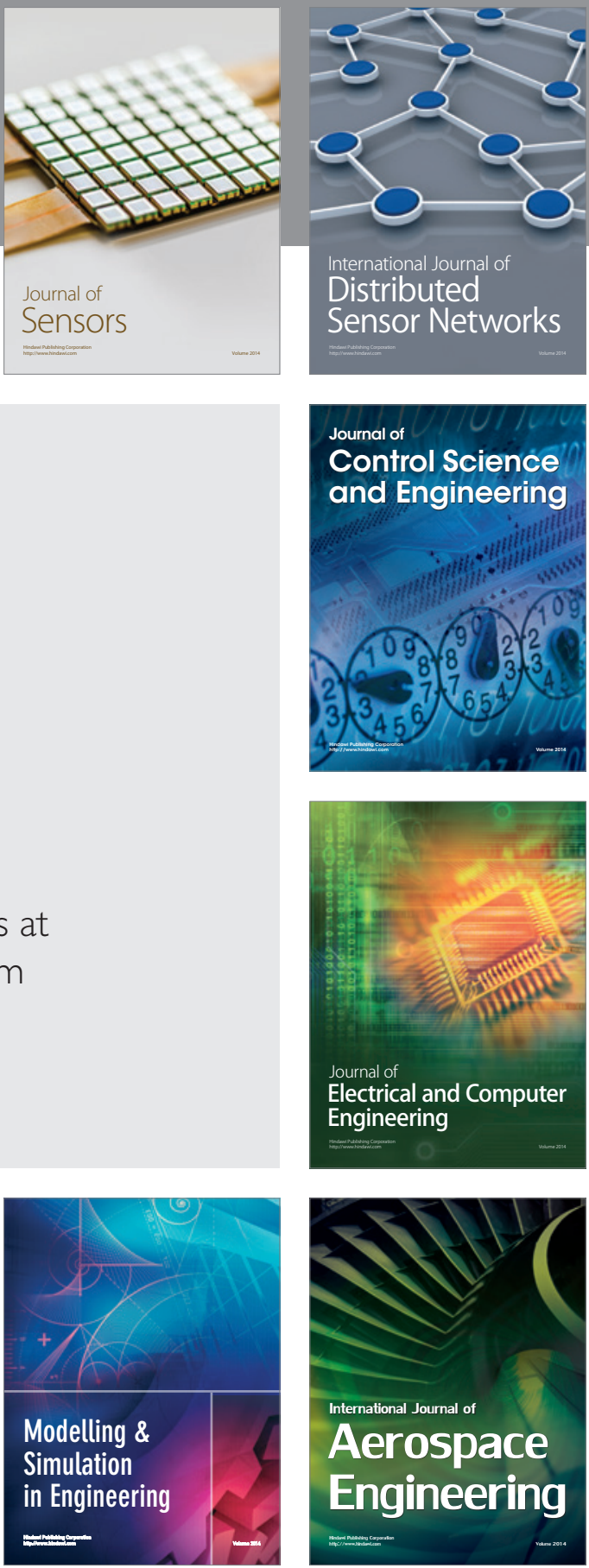

Journal of

Control Science

and Engineering
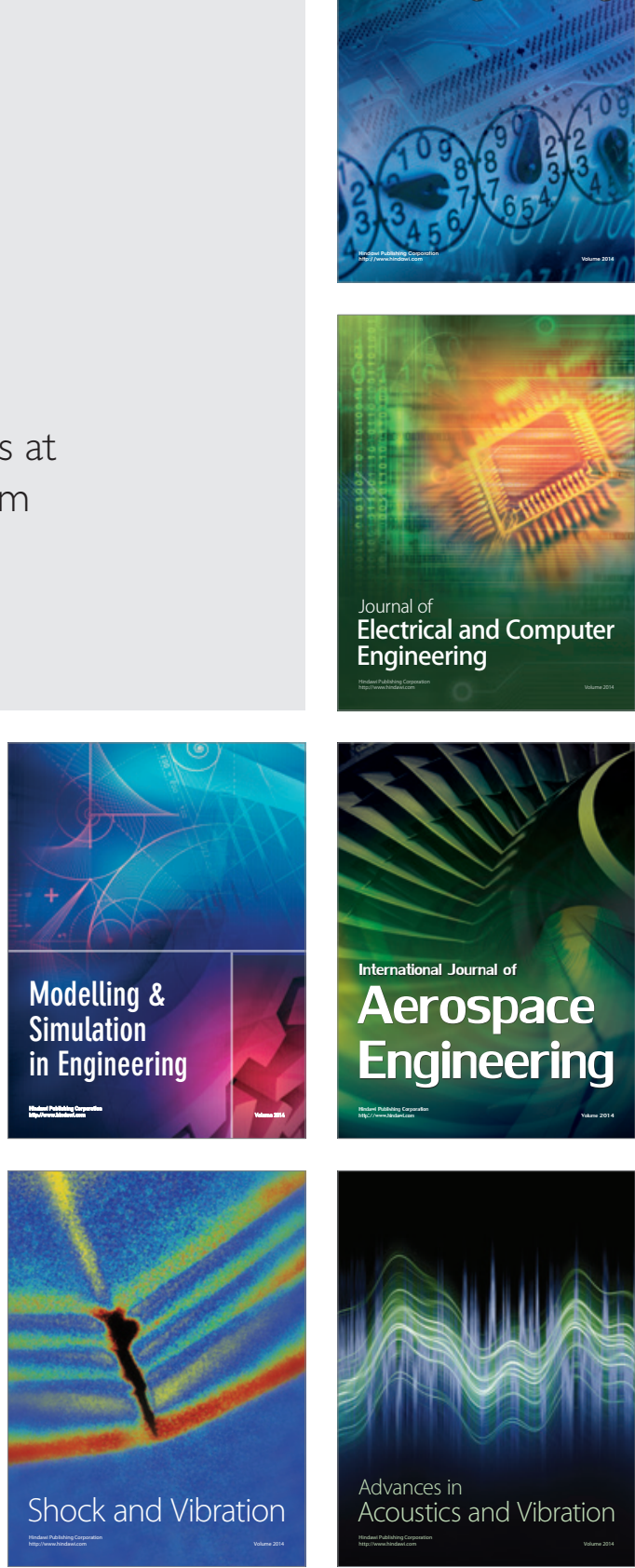